\title{
Factorization theorem for product Hardy spaces
}

\author{
by \\ Wengu Chen (Beijing), Yongsheng Han (Auburn, AL) and \\ ChangXing Miao (Beijing)
}

\begin{abstract}
We extend the well known factorization theorems on the unit disk to product Hardy spaces, which generalizes the previous results obtained by Coifman, Rochberg and Weiss. The basic tools are the boundedness of a certain bilinear form on $\mathbb{R}_{+}^{2} \times \mathbb{R}_{+}^{2}$ and the characterization of $\mathrm{BMO}\left(\mathbb{R}_{+}^{2} \times \mathbb{R}_{+}^{2}\right)$ recently obtained by Ferguson, Lacey and Sadosky.
\end{abstract}

1. Introduction. The purpose of this note is to extend some well known factorization theorems to product Hardy spaces. We begin by recalling the known results. In the one-dimensional case, let $F$ be holomorphic in $\{|z|<1\}$ and satisfy

$$
\sup _{0<r<1} \int_{0}^{2 \pi}\left|F\left(r e^{i \theta}\right)\right| d \theta \leq 1 .
$$

It is well known that $F=G_{1} G_{2}$ with $G_{1}$ and $G_{2}$ holomorphic and

$$
\left\|G_{i}\right\|_{H^{2}}^{2}=\sup _{0<r<1} \int_{0}^{2 \pi}\left|G_{i}\left(r e^{i \theta}\right)\right|^{2} d \theta \leq 1, \quad i=1,2 .
$$

Coifman, Rochberg and Weiss extended this theorem to Hardy spaces in several variables. To be precise, the Hardy space $H^{1}\left(\mathbb{R}^{n}\right)$ used in [3] is defined by

$$
\|f\|_{H^{1}\left(\mathbb{R}^{n}\right)}=\|f\|_{1}+\sum_{j=1}^{n}\left\|R_{j}(f)\right\|_{1}
$$

where $R_{j}(f), j=1, \ldots, n$, are the Riesz transforms of $f$. In [3] it was proved that for every $f \in H^{1}\left(\mathbb{R}^{n}\right)$,

2000 Mathematics Subject Classification: Primary 42B20; Secondary 42B30, 42B25.

Key words and phrases: product Hardy space, factorization theorem, bilinear form.

The first author was supported by NNSF (No. 10371080) of China and Scientific Research Foundation for Returned Overseas Chinese Scholars. 


$$
f=\sum_{i=1}^{\infty} \sum_{j=1}^{n}\left[g_{i}^{j} R_{j}\left(h_{i}^{j}\right)+h_{i}^{j} R_{j}\left(g_{i}^{j}\right)\right]
$$

for some $\left\{g_{i}^{j}, h_{i}^{j}\right\} \subset L^{2}\left(\mathbb{R}^{n}\right)$, and moreover, there exist two constants $c_{1}$ and $c_{2}$ such that

$$
c_{1} \sum_{i=1}^{\infty} \sum_{j=1}^{n}\left\|g_{i}^{j}\right\|_{2}\left\|h_{i}^{j}\right\|_{2} \leq\|f\|_{H^{1}} \leq c_{2} \sum_{i=1}^{\infty} \sum_{j=1}^{n}\left\|g_{i}^{j}\right\|_{2}\left\|h_{i}^{j}\right\|_{2} .
$$

The basic idea of the proof in [3] was the duality between $H^{1}$ and BMO and a new characterization of BMO in terms of the $L^{2}$ boundedness of the commutator of a singular integral operator and a multiplication operator. It is worth pointing out that $(\mathrm{VMO})^{*}=H^{1}$ was used. See $[3,(2.4)$, p. 614].

Uchiyama [7] extended the result of Coifman, Rochberg and Weiss to spaces of homogeneous type. The new idea used in [7] is the boundedness of a certain bilinear form and the atomic decomposition of $H^{p}$ on spaces of homogeneous type.

More recently, Ferguson, Sadosky and Lacey (see [6] and [5]) gave a new characterization of BMO on the product space $\mathbb{R}_{+}^{2} \times \mathbb{R}_{+}^{2}$ in terms of the nested commutator determined by a function $b$ and the one-dimensional Hilbert transforms $H_{j}, j=1,2$, in the $j$ th variable. See [5] for the details.

In this note, we extend the Coifman, Rochberg and Weiss factorization theorem to $H^{1}\left(\mathbb{R}_{+}^{2} \times \mathbb{R}_{+}^{2}\right)$. Since $H^{1}\left(\mathbb{R}_{+}^{2} \times \mathbb{R}_{+}^{2}\right)$ is not a simple generalization of the classical $H^{1}\left(\mathbb{R}^{2}\right)$, so far no characterization of the predual space of $H^{1}\left(\mathbb{R}_{+}^{2} \times \mathbb{R}_{+}^{2}\right)$ is known, and the atomic decomposition of $H^{p}$ used by Uchiyama does not work for product $H^{p}$ spaces either. The basic idea in this note is to introduce a certain bilinear form on $\mathbb{R}_{+}^{2} \times \mathbb{R}_{+}^{2}$ in terms of the Hilbert transforms $H_{j}, j=1,2$, and to prove its boundedness on $H^{1}\left(\mathbb{R}_{+}^{2} \times \mathbb{R}_{+}^{2}\right)$. Finally, this result together with the characterization of $\mathrm{BMO}\left(\mathbb{R}_{+}^{2} \times \mathbb{R}_{+}^{2}\right)$ obtained in [5] gives the factorization theorem for $H^{1}\left(\mathbb{R}_{+}^{2} \times \mathbb{R}_{+}^{2}\right)$.

We begin by recalling the definition of Hardy spaces in product domains (see [1], [2] and [4]). Let $\psi^{(i)} \in C_{0}^{\infty}(\mathbb{R}), i=1,2$, be supported in the unit interval, with $\int_{\mathbb{R}} \psi^{(i)}(x) d x=1$. Set $\psi_{t_{i}}^{(i)}\left(x_{i}\right)=t_{i}^{-1} \psi^{(i)}\left(x_{i} / t_{i}\right), i=1,2$, for $t>0$. For $f \in L^{1}(\mathbb{R} \times \mathbb{R})$, let

$$
f^{*}\left(x_{1}, x_{2}\right)=\sup _{t_{1}, t_{2}>0}\left|\iint f\left(y_{1}, y_{2}\right) \psi_{t_{1}}^{(1)}\left(x_{1}-y_{1}\right) \psi_{t_{2}}^{(2)}\left(x_{2}-y_{2}\right) d y_{1} d y_{2}\right| .
$$

We say $f \in H^{1}(\mathbb{R} \times \mathbb{R})$ if $f^{*} \in L^{1}(\mathbb{R} \times \mathbb{R})$ and we define

$$
\|f\|_{H^{1}(\mathbb{R} \times \mathbb{R})}=\left\|f^{*}\right\|_{L^{1}(\mathbb{R} \times \mathbb{R})} .
$$

The main results of this note are the following theorems. 
Theorem 1. If $1<q<\infty, g \in L^{q}(\mathbb{R} \times \mathbb{R})$ and $h \in L^{q^{\prime}}(\mathbb{R} \times \mathbb{R})$, $1 / q+1 / q^{\prime}=1$, then

$$
\|D(g, h)\|_{H^{1}(\mathbb{R} \times \mathbb{R})} \leq c\|g\|_{L^{q}(\mathbb{R} \times \mathbb{R})}\|h\|_{L^{q^{\prime}(\mathbb{R} \times \mathbb{R})}},
$$

where

$$
\begin{aligned}
& D(g, h)\left(x_{1}, x_{2}\right) \\
& \quad=h\left(H_{2}\left(H_{1}(g)\right)\right)+H_{1}(g) H_{2}(h)+H_{2}(g) H_{1}(h)+g\left(H_{2}\left(H_{1}(h)\right)\right),
\end{aligned}
$$

and $c$ is a positive constant depending only on $q$.

TheOREM 2. If $f \in H^{1}(\mathbb{R} \times \mathbb{R})$, then there exist some $\left\{g_{i}\right\} \subset L^{2}(\mathbb{R} \times \mathbb{R})$ and $\left\{h_{i}\right\} \subset L^{2}(\mathbb{R} \times \mathbb{R})$ and two positive constants $c_{1}$ and $c_{2}$ such that

$$
f=\sum_{i=1}^{\infty} D\left(g_{i}, h_{i}\right)
$$

and

$$
c_{1} \sum_{i=1}^{\infty}\left\|g_{i}\right\|_{2}\left\|h_{i}\right\|_{2} \leq\|f\|_{H^{1}} \leq c_{2} \sum_{i=1}^{\infty}\left\|g_{i}\right\|_{2}\left\|h_{i}\right\|_{2}
$$

2. Proofs. To prove the main results of this note, we need other definitions and notation. For $f \in L_{\text {loc }}^{1}(\mathbb{R} \times \mathbb{R})$, the strong maximal function is defined by

$$
\mathcal{M} f\left(x_{1}, x_{2}\right)=\sup _{\left(x_{1}, x_{2}\right) \in R} \frac{1}{|R|} \iint_{R}\left|f\left(y_{1}, y_{2}\right)\right| d y_{1} d y_{2},
$$

where the supremum is taken over all rectangles $R$ which contain $\left(x_{1}, x_{2}\right)$. For $0<r<\infty, 0<\varepsilon<1$, we define

$$
\begin{aligned}
M_{r}^{(1)} f\left(x_{1}, x_{2}\right) & =\sup _{t_{1}>0}\left(\frac{1}{t_{1}} \int_{\left|y_{1}-x_{1}\right|<t_{1}}\left|f\left(y_{1}, x_{2}\right)\right|^{r} d y_{1}\right)^{1 / r}, \\
I_{\varepsilon}^{(1)} f\left(x_{1}, x_{2}\right) & =\text { p.v. } \int \frac{f\left(y_{1}, x_{2}\right)}{\left|x_{1}-y_{1}\right|^{1-\varepsilon}} d y_{1} .
\end{aligned}
$$

$M_{r}^{(2)}$ and $I_{\varepsilon}^{(2)}$ are defined in a similar way. When $r=1$, we write $M^{(j)}$ for $M_{1}^{(j)}, j=1,2$. Finally, we define the maximal operators of the Hilbert transforms $H_{1}$ and $H_{2}$ in the first and second variable respectively by

$$
\begin{aligned}
& H_{1}^{*} f\left(x_{1}, x_{2}\right)=\sup _{t_{1}>0}\left|\int_{\left|y_{1}-x_{1}\right|>t_{1}} \frac{f\left(y_{1}, x_{2}\right)}{x_{1}-y_{1}} d y_{1}\right|, \\
& H_{2}^{*} f\left(x_{1}, x_{2}\right)=\sup _{t_{2}>0}\left|\int_{\left|y_{2}-x_{2}\right|>t_{2}} \frac{f\left(x_{1}, y_{2}\right)}{x_{2}-y_{2}} d y_{2}\right| .
\end{aligned}
$$


Proof of Theorem 1. Fix $\left(x_{1}, x_{2}\right) \in \mathbb{R} \times \mathbb{R}$ and let $t_{1}, t_{2}>0$. Then

$$
\begin{aligned}
\iint D( & g, h)\left(y_{1}, y_{2}\right) \psi_{t_{1}}^{(1)}\left(x_{1}-y_{1}\right) \psi_{t_{2}}^{(2)}\left(x_{2}-y_{2}\right) d y_{1} d y_{2} \\
= & \iint \frac{g\left(z_{1}, z_{2}\right)}{\left(y_{1}-z_{1}\right)\left(y_{2}-z_{2}\right)}\left[\psi_{t_{1}}^{(1)}\left(x_{1}-z_{1}\right)-\psi_{t_{1}}^{(1)}\left(x_{1}-y_{1}\right)\right] \\
& \times\left[\psi_{t_{2}}^{(2)}\left(x_{2}-z_{2}\right)-\psi_{t_{2}}^{(2)}\left(x_{2}-y_{2}\right)\right] d z_{1} d z_{2} h\left(y_{1}, y_{2}\right) d y_{1} d y_{2} \\
= & \iint \eta\left(y_{1}, y_{2}, z_{1}, z_{2}\right) d z_{1} d z_{2} h\left(y_{1}, y_{2}\right) d y_{1} d y_{2},
\end{aligned}
$$

where

$$
\begin{aligned}
\eta\left(y_{1}, y_{2}, z_{1}, z_{2}\right)= & \frac{g\left(z_{1}, z_{2}\right)}{\left(y_{1}-z_{1}\right)\left(y_{2}-z_{2}\right)}\left[\psi_{t_{1}}^{(1)}\left(x_{1}-z_{1}\right)-\psi_{t_{1}}^{(1)}\left(x_{1}-y_{1}\right)\right] \\
& \times\left[\psi_{t_{2}}^{(2)}\left(x_{2}-z_{2}\right)-\psi_{t_{2}}^{(2)}\left(x_{2}-y_{2}\right)\right] .
\end{aligned}
$$

CASE I: $\left|x_{1}-y_{1}\right|>4 t_{1},\left|x_{2}-y_{2}\right|>4 t_{2}$. Define

$$
\Omega_{1}=\left\{\left(y_{1}, y_{2}\right) \in \mathbb{R} \times \mathbb{R}:\left|x_{1}-y_{1}\right|>4 t_{1},\left|x_{2}-y_{2}\right|>4 t_{2}\right\} .
$$

Then $\psi_{t_{1}}^{(1)}\left(x_{1}-y_{1}\right)=\psi_{t_{2}}^{(2)}\left(x_{2}-y_{2}\right)=0$ when $\left(y_{1}, y_{2}\right) \in \Omega_{1}$, and

$=\iint\left[\frac{1}{y_{1}-z_{1}}-\frac{1}{y_{1}-x_{1}}\right]\left[\frac{1}{y_{2}-z_{2}}-\frac{1}{y_{2}-x_{2}}\right] \psi_{t_{1}}^{(1)}\left(x_{1}-z_{1}\right) \psi_{t_{2}}^{(2)}\left(x_{2}-z_{2}\right)$

$\times g\left(z_{1}, z_{2}\right) d z_{1} d z_{2}$

$+\frac{1}{y_{1}-x_{1}} \iint\left[\frac{1}{y_{2}-z_{2}}-\frac{1}{y_{2}-x_{2}}\right] \psi_{t_{1}}^{(1)}\left(x_{1}-z_{1}\right) \psi_{t_{2}}^{(2)}\left(x_{2}-z_{2}\right) g\left(z_{1}, z_{2}\right) d z_{1} d z_{2}$

$+\frac{1}{y_{2}-x_{2}} \iint\left[\frac{1}{y_{1}-z_{1}}-\frac{1}{y_{1}-x_{1}}\right] \psi_{t_{1}}^{(1)}\left(x_{1}-z_{1}\right) \psi_{t_{2}}^{(2)}\left(x_{2}-z_{2}\right) g\left(z_{1}, z_{2}\right) d z_{1} d z_{2}$

$+\frac{1}{\left(y_{1}-x_{1}\right)\left(y_{2}-x_{2}\right)} \iint \psi_{t_{1}}^{(1)}\left(x_{1}-z_{1}\right) \psi_{t_{2}}^{(2)}\left(x_{2}-z_{2}\right) g\left(z_{1}, z_{2}\right) d z_{1} d z_{2}$

$=\eta_{1}\left(y_{1}, y_{2}\right)+\eta_{2}\left(y_{1}, y_{2}\right)+\eta_{3}\left(y_{1}, y_{2}\right)+\eta_{4}\left(y_{1}, y_{2}\right)$.

If $\psi_{t_{1}}^{(1)}\left(x_{1}-z_{1}\right) \neq 0$, then $\left|x_{1}-z_{1}\right|<\left|x_{1}-y_{1}\right| / 2$. Similarly, if $\psi_{t_{2}}^{(2)}\left(x_{2}-z_{2}\right) \neq 0$, then $\left|x_{2}-z_{2}\right|<\left|x_{2}-y_{2}\right| / 2$. Hence

$$
\left|\eta_{1}\left(y_{1}, y_{2}\right)\right| \leq c \frac{t_{1}}{\left(t_{1}+\left|y_{1}-x_{1}\right|\right)^{2}} \frac{t_{2}}{\left(t_{2}+\left|y_{2}-x_{2}\right|\right)^{2}} \mathcal{M g}\left(x_{1}, x_{2}\right) .
$$

So,

$$
\left|\iint_{\Omega_{1}} \eta_{1}\left(y_{1}, y_{2}\right) h\left(y_{1}, y_{2}\right) d y_{1} d y_{2}\right| \leq c \mathcal{M} g\left(x_{1}, x_{2}\right) \mathcal{M} h\left(x_{1}, x_{2}\right)
$$


For the other three terms $\eta_{2}\left(y_{1}, y_{2}\right), \eta_{3}\left(y_{1}, y_{2}\right)$ and $\eta_{4}\left(y_{1}, y_{2}\right)$, by integrating against $h\left(y_{1}, y_{2}\right)$ with respect to $y_{1}$ and $y_{2}$ directly, we obtain

$$
\begin{aligned}
\mid \iint_{\Omega_{1}} \eta_{2}\left(y_{1}, y_{2}\right) & h\left(y_{1}, y_{2}\right) d y_{1} d y_{2} \mid \\
& \leq c \mathcal{M g}\left(x_{1}, x_{2}\right) \int\left|H_{1}^{*} h\left(x_{1}, y_{2}\right)\right| \frac{t_{2}}{\left(t_{2}+\left|y_{2}-x_{2}\right|\right)^{2}} d y_{2} \\
& \leq \operatorname{cMg} g\left(x_{1}, x_{2}\right) M^{(2)}\left(H_{1}^{*} h\right)\left(x_{1}, x_{2}\right),
\end{aligned}
$$

$$
\begin{aligned}
\mid \iint_{\Omega_{1}} \eta_{3}\left(y_{1}, y_{2}\right) & h\left(y_{1}, y_{2}\right) d y_{1} d y_{2} \mid \\
& \leq c \mathcal{M g} g\left(x_{1}, x_{2}\right) \int\left|H_{2}^{*} h\left(y_{1}, x_{2}\right)\right| \frac{t_{1}}{\left(t_{1}+\left|y_{1}-x_{1}\right|\right)^{2}} d y_{1} \\
& \leq c \mathcal{M g}\left(x_{1}, x_{2}\right) M^{(1)}\left(H_{2}^{*} h\right)\left(x_{1}, x_{2}\right),
\end{aligned}
$$

and

$$
\left|\iint_{\Omega_{1}} \eta_{4}\left(y_{1}, y_{2}\right) h\left(y_{1}, y_{2}\right) d y_{1} d y_{2}\right| \leq c \mathcal{M g}\left(x_{1}, x_{2}\right) H_{1}^{*}\left(H_{2}^{*} h\right)\left(x_{1}, x_{2}\right) .
$$

CASE II: $\left|x_{1}-y_{1}\right| \leq 4 t_{1},\left|x_{2}-y_{2}\right| \leq 4 t_{2}$. Define

$$
\Omega_{2}=\left\{\left(y_{1}, y_{2}\right) \in \mathbb{R} \times \mathbb{R}:\left|x_{1}-y_{1}\right| \leq 4 t_{1},\left|x_{2}-y_{2}\right| \leq 4 t_{2}\right\}
$$

and let $\phi \in C^{\infty}(0, \infty)$ be a fixed nonnegative function such that $\phi(t)=0$ on $(0,1 / 2), \phi(t)=1$ on $(1, \infty)$ and $|d \phi / d t|<3$. Set $\phi^{\prime}=1-\phi$. Then

$$
\begin{aligned}
& \iint \eta\left(y_{1}, y_{2}, z_{1}, z_{2}\right) d z_{1} d z_{2} \\
= & \iint \frac{g\left(z_{1}, z_{2}\right)}{\left(y_{1}-z_{1}\right)\left(y_{2}-z_{2}\right)}\left[\psi_{t_{1}}^{(1)}\left(x_{1}-z_{1}\right)-\psi_{t_{1}}^{(1)}\left(x_{1}-y_{1}\right)\right] \\
& \times\left[\psi_{t_{2}}^{(2)}\left(x_{2}-z_{2}\right)-\psi_{t_{2}}^{(2)}\left(x_{2}-y_{2}\right)\right]\left[\phi\left(\left|x_{1}-z_{1}\right| / 16 t_{1}\right)+\phi^{\prime}\left(\left|x_{1}-z_{1}\right| / 16 t_{1}\right)\right] \\
& \times\left[\phi\left(\left|x_{2}-z_{2}\right| / 16 t_{2}\right)+\phi^{\prime}\left(\left|x_{2}-z_{2}\right| / 16 t_{2}\right)\right] d z_{1} d z_{2} \\
= & \psi_{t_{1}}^{(1)}\left(x_{1}-y_{1}\right) \psi_{t_{2}}^{(2)}\left(x_{2}-y_{2}\right) \iint\left[\frac{1}{y_{1}-z_{1}}-\frac{1}{x_{1}-z_{1}}\right]\left[\frac{1}{y_{2}-z_{2}}-\frac{1}{x_{2}-z_{2}}\right] \\
& \times \phi\left(\left|x_{1}-z_{1}\right| / 16 t_{1}\right) \phi\left(\left|x_{2}-z_{2}\right| / 16 t_{2}\right) g\left(z_{1}, z_{2}\right) d z_{1} d z_{2} \\
& +\psi_{t_{1}}^{(1)}\left(x_{1}-y_{1}\right) \psi_{t_{2}}^{(2)}\left(x_{2}-y_{2}\right) \iint \frac{1}{x_{1}-z_{1}}\left[\frac{1}{y_{2}-z_{2}}-\frac{1}{x_{2}-z_{2}}\right] \\
& \times \phi\left(\left|x_{1}-z_{1}\right| / 16 t_{1}\right) \phi\left(\left|x_{2}-z_{2}\right| / 16 t_{2}\right) g\left(z_{1}, z_{2}\right) d z_{1} d z_{2} \\
& +\psi_{t_{1}}^{(1)}\left(x_{1}-y_{1}\right) \psi_{t_{2}}^{(2)}\left(x_{2}-y_{2}\right) \iint \frac{1}{x_{2}-z_{2}}\left[\frac{1}{y_{1}-z_{1}}-\frac{1}{x_{1}-z_{1}}\right] \\
& \times \phi\left(\left|x_{1}-z_{1}\right| / 16 t_{1}\right) \phi\left(\left|x_{2}-z_{2}\right| / 16 t_{2}\right) g\left(z_{1}, z_{2}\right) d z_{1} d z_{2} \\
& +\psi_{t_{1}}^{(1)}\left(x_{1}-y_{1}\right) \psi_{t_{2}}^{(2)}\left(x_{2}-y_{2}\right) \iint \frac{1}{\left(x_{1}-z_{1}\right)\left(x_{2}-z_{2}\right)}
\end{aligned}
$$




$$
\begin{aligned}
& \times \phi\left(\left|x_{1}-z_{1}\right| / 16 t_{1}\right) \phi\left(\left|x_{2}-z_{2}\right| / 16 t_{2}\right) g\left(z_{1}, z_{2}\right) d z_{1} d z_{2} \\
& +\psi_{t_{1}}^{(1)}\left(x_{1}-y_{1}\right) \iint\left[\frac{1}{y_{1}-z_{1}}-\frac{1}{x_{1}-z_{1}}\right] \frac{1}{y_{2}-z_{2}}\left[\psi_{t_{2}}^{(2)}\left(x_{2}-y_{2}\right)-\psi_{t_{2}}^{(2)}\left(x_{2}-z_{2}\right)\right] \\
& \times \phi\left(\left|x_{1}-z_{1}\right| / 16 t_{1}\right) \phi^{\prime}\left(\left|x_{2}-z_{2}\right| / 16 t_{2}\right) g\left(z_{1}, z_{2}\right) d z_{1} d z_{2} \\
& +\psi_{t_{1}}^{(1)}\left(x_{1}-y_{1}\right) \iint \frac{1}{\left(x_{1}-z_{1}\right)\left(y_{2}-z_{2}\right)}\left[\psi_{t_{2}}^{(2)}\left(x_{2}-y_{2}\right)-\psi_{t_{2}}^{(2)}\left(x_{2}-z_{2}\right)\right] \\
& \times \phi\left(\left|x_{1}-z_{1}\right| / 16 t_{1}\right) \phi^{\prime}\left(\left|x_{2}-z_{2}\right| / 16 t_{2}\right) g\left(z_{1}, z_{2}\right) d z_{1} d z_{2} \\
& +\psi_{t_{2}}^{(2)}\left(x_{2}-y_{2}\right) \iint\left[\frac{1}{y_{2}-z_{2}}-\frac{1}{x_{2}-z_{2}}\right] \frac{1}{y_{1}-z_{1}}\left[\psi_{t_{1}}^{(1)}\left(x_{1}-y_{1}\right)-\psi_{t_{1}}^{(1)}\left(x_{1}-z_{1}\right)\right] \\
& \times \phi^{\prime}\left(\left|x_{1}-z_{1}\right| / 16 t_{1}\right) \phi\left(\left|x_{2}-z_{2}\right| / 16 t_{2}\right) g\left(z_{1}, z_{2}\right) d z_{1} d z_{2} \\
& +\psi_{t_{2}}^{(2)}\left(x_{2}-y_{2}\right) \iint \frac{1}{\left(y_{1}-z_{1}\right)\left(x_{2}-z_{2}\right)}\left[\psi_{t_{1}}^{(1)}\left(x_{1}-y_{1}\right)-\psi_{t_{1}}^{(1)}\left(x_{1}-z_{1}\right)\right] \\
& \times \phi^{\prime}\left(\left|x_{1}-z_{1}\right| / 16 t_{1}\right) \phi\left(\left|x_{2}-z_{2}\right| / 16 t_{2}\right) g\left(z_{1}, z_{2}\right) d z_{1} d z_{2} \\
& +\iint \frac{1}{\left(y_{1}-z_{1}\right)\left(y_{2}-z_{2}\right)}\left[\psi_{t_{1}}^{(1)}\left(x_{1}-z_{1}\right)-\psi_{t_{1}}^{(1)}\left(x_{1}-y_{1}\right)\right] g\left(z_{1}, z_{2}\right) \\
& \left.\times\left[\psi_{t_{2}}^{(2)}\left(x_{2}-z_{2}\right)-\psi_{t_{2}}^{(2)}\left(x_{2}-y_{2}\right)\right] \phi^{\prime}\left(\left|x_{1}-z_{1}\right| / 16 t_{1}\right) \phi^{\prime}\left(\left|x_{2}-z_{2}\right| / 16 t_{2}\right)\right] d z_{1} d z_{2} \\
& =\sum_{j=5} \eta_{j}\left(y_{1}, y_{2}\right) .
\end{aligned}
$$

If $\phi\left(\left|x_{1}-z_{1}\right| / 16 t_{1}\right) \neq 0$, then $\left|x_{1}-z_{1}\right| \geq 8 t_{1}$, hence $\left|x_{1}-y_{1}\right|<\left|x_{1}-z_{1}\right| / 2$. Similarly, if $\phi\left(\left|x_{2}-z_{2}\right| / 16 t_{2}\right) \neq 0$, then $\left|x_{2}-z_{2}\right| \geq 8 t_{2}$ and $\left|x_{2}-y_{2}\right|<$ $2\left|x_{2}-z_{2}\right| / 2$. Hence,

$$
\begin{aligned}
\left|\eta_{5}\left(y_{1}, y_{2}\right)\right| \leq & c\left|\psi_{t_{1}}^{(1)}\left(x_{1}-y_{1}\right) \psi_{t_{2}}^{(2)}\left(x_{2}-y_{2}\right)\right| \iint \frac{\left|y_{1}-x_{1}\right|}{\left|x_{1}-z_{1}\right|^{2}} \frac{\left|y_{2}-x_{2}\right|}{\left|x_{2}-z_{2}\right|^{2}} \\
& \times \phi\left(\left|x_{1}-z_{1}\right| / 16 t_{1}\right) \phi\left(\left|x_{2}-z_{2}\right| / 16 t_{2}\right)\left|g\left(z_{1}, z_{2}\right)\right| d z_{1} d z_{2} \\
\leq & c\left|\psi_{t_{1}}^{(1)}\left(x_{1}-y_{1}\right) \psi_{t_{2}}^{(2)}\left(x_{2}-y_{2}\right)\right| \mathcal{M} g\left(x_{1}, x_{2}\right) .
\end{aligned}
$$

To estimate the other terms, define a variant of the Hilbert transform in the first variable,

$$
\widetilde{H}_{1} f\left(x_{1}, x_{2}\right)=\lim _{t_{1} \rightarrow 0} \int \frac{f\left(z_{1}, x_{2}\right)}{x_{1}-z_{1}} \phi\left(\left|x_{1}-z_{1}\right| / 16 t_{1}\right) d z_{1},
$$

and its maximal operator

$$
\widetilde{H}_{1}^{*} f\left(x_{1}, x_{2}\right)=\sup _{t_{1}>0}\left|\int \frac{f\left(z_{1}, x_{2}\right)}{x_{1}-z_{1}} \phi\left(\left|x_{1}-z_{1}\right| / 16 t_{1}\right) d z_{1}\right| .
$$

Similarly, we define a variant of the Hilbert transform in the second variable,

$$
\widetilde{H}_{2} f\left(x_{1}, x_{2}\right)=\lim _{t_{2} \rightarrow 0} \int \frac{f\left(x_{1}, z_{2}\right)}{x_{2}-z_{2}} \phi\left(\left|x_{2}-z_{2}\right| / 16 t_{2}\right) d z_{2},
$$


and its maximal operator

$$
\widetilde{H}_{2}^{*} f\left(x_{1}, x_{2}\right)=\sup _{t_{2}>0}\left|\int \frac{f\left(x_{1}, z_{2}\right)}{x_{2}-z_{2}} \phi\left(\left|x_{2}-z_{2}\right| / 16 t_{2}\right) d z_{2}\right| .
$$

By standard arguments, it is easy to prove that $\widetilde{H}_{j}$ and $\widetilde{H}_{j}^{*}$ are $L^{p} \rightarrow L^{p}$ bounded for all $1<p<\infty, j=1,2$.

By means of $\widetilde{H}_{1}$ and $\widetilde{H}_{1}^{*}$, we obtain an estimate for $\eta_{6}\left(y_{1}, y_{2}\right)$ :

$$
\left|\eta_{6}\left(y_{1}, y_{2}\right)\right| \leq c\left|\psi_{t_{1}}^{(1)}\left(x_{1}-y_{1}\right) \psi_{t_{2}}^{(2)}\left(x_{2}-y_{2}\right)\right| M^{(2)}\left(\widetilde{H}_{1}^{*} g\right)\left(x_{1}, x_{2}\right) .
$$

The estimates for $\eta_{7}\left(y_{1}, y_{2}\right)$ and $\eta_{8}\left(y_{1}, y_{2}\right)$ are similar:

$$
\begin{aligned}
& \left|\eta_{7}\left(y_{1}, y_{2}\right)\right| \leq c\left|\psi_{t_{1}}^{(1)}\left(x_{1}-y_{1}\right) \psi_{t_{2}}^{(2)}\left(x_{2}-y_{2}\right)\right| M^{(1)}\left(\widetilde{H}_{2}^{*} g\right)\left(x_{1}, x_{2}\right), \\
& \left|\eta_{8}\left(y_{1}, y_{2}\right)\right| \leq c\left|\psi_{t_{1}}^{(1)}\left(x_{1}-y_{1}\right) \psi_{t_{2}}^{(2)}\left(x_{2}-y_{2}\right)\right| \widetilde{H}_{1}^{*}\left(\widetilde{H}_{2}^{*} g\right)\left(x_{1}, x_{2}\right) .
\end{aligned}
$$

To estimate the remaining terms, we choose $\varepsilon \in(0,1)$ such that

$$
\begin{aligned}
& \left|\psi_{t_{1}}^{(1)}\left(x_{1}-z_{1}\right)-\psi_{t_{1}}^{(1)}\left(x_{1}-y_{1}\right)\right| \leq c\left|y_{1}-z_{1}\right|^{\varepsilon} / t_{1}^{1+\varepsilon}, \\
& \left|\psi_{t_{2}}^{(2)}\left(x_{2}-z_{2}\right)-\psi_{t_{2}}^{(2)}\left(x_{2}-y_{2}\right)\right| \leq c\left|y_{2}-z_{2}\right|^{\varepsilon} / t_{2}^{1+\varepsilon} .
\end{aligned}
$$

Then

$$
\begin{aligned}
\left|\eta_{9}\left(y_{1}, y_{2}\right)\right| & \leq \frac{c}{t_{2}^{1+\varepsilon}}\left|\psi_{t_{1}}^{(1)}\left(x_{1}-y_{1}\right)\right| I_{\varepsilon}^{(2)}\left(M^{(1)}(g) \phi^{\prime}\left(\left|x_{2}-\cdot\right| / 16 t_{2}\right)\right)\left(x_{1}, y_{2}\right), \\
\left|\eta_{10}\left(y_{1}, y_{2}\right)\right| & \leq \frac{c}{t_{2}^{1+\varepsilon}}\left|\psi_{t_{1}}^{(1)}\left(x_{1}-y_{1}\right)\right| I_{\varepsilon}^{(2)}\left(\widetilde{H}_{1}^{*}(g) \phi^{\prime}\left(\left|x_{2}-\cdot\right| / 16 t_{2}\right)\right)\left(x_{1}, y_{2}\right) .
\end{aligned}
$$

The estimates for the last three terms are similar:

$\left|\eta_{11}\left(y_{1}, y_{2}\right)\right| \leq \frac{c}{t_{1}^{1+\varepsilon}}\left|\psi_{t_{2}}^{(2)}\left(x_{2}-y_{2}\right)\right| I_{\varepsilon}^{(1)}\left(M^{(2)}(g) \phi^{\prime}\left(\left|x_{1}-\cdot\right| / 16 t_{1}\right)\right)\left(y_{1}, x_{2}\right)$,

$\left|\eta_{12}\left(y_{1}, y_{2}\right)\right| \leq \frac{c}{t_{1}^{1+\varepsilon}}\left|\psi_{t_{2}}^{(2)}\left(x_{2}-y_{2}\right)\right| I_{\varepsilon}^{(1)}\left(\widetilde{H}_{2}^{*}(g) \phi^{\prime}\left(\left|x_{1}-\cdot\right| / 16 t_{1}\right)\right)\left(y_{1}, x_{2}\right)$,

$\left|\eta_{13}\left(y_{1}, y_{2}\right)\right| \leq \frac{c}{t_{1}^{1+\varepsilon} t_{2}^{1+\varepsilon}} I_{\varepsilon}^{(1)}\left(I_{\varepsilon}^{(2)}\left(|g| \phi^{\prime}\left(\left|x_{2}-\cdot\right| / 16 t_{2}\right)\right) \phi^{\prime}\left(\left|x_{1}-\cdot\right| / 16 t_{1}\right)\right)\left(y_{1}, y_{2}\right)$.

Integrating $\eta_{j}\left(y_{1}, y_{2}\right)$ against $h\left(y_{1}, y_{2}\right)$ over $\Omega_{2}$ with respect to $y_{1}$ and $y_{2}$ together with the above estimates for $\eta_{j}\left(y_{1}, y_{2}\right), j=5,6, \ldots, 13$, we obtain estimates for the first four terms:

$$
\begin{aligned}
&\left|\int_{*} \eta_{5}\left(y_{1}, y_{2}\right) h\left(y_{1}, y_{2}\right) d y_{1} d y_{2}\right| \leq c \mathcal{M} g\left(x_{1}, x_{2}\right) \mathcal{M} h\left(x_{1}, x_{2}\right), \\
&\left|\int_{*} \eta_{6}\left(y_{1}, y_{2}\right) h\left(y_{1}, y_{2}\right) d y_{1} d y_{2}\right| \leq c M^{(2)}\left(\widetilde{H}_{1}^{*} g\right)\left(x_{1}, x_{2}\right) \mathcal{M} h\left(x_{1}, x_{2}\right), \\
&\left|\int_{*} \eta_{7}\left(y_{1}, y_{2}\right) h\left(y_{1}, y_{2}\right) d y_{1} d y_{2}\right| \leq c M^{(1)}\left(\widetilde{H}_{2}^{*} g\right)\left(x_{1}, x_{2}\right) \mathcal{M} h\left(x_{1}, x_{2}\right), \\
&\left|\int_{*} \eta_{8}\left(y_{1}, y_{2}\right) h\left(y_{1}, y_{2}\right) d y_{1} d y_{2}\right| \leq c \widetilde{H}_{1}^{*}\left(\widetilde{H}_{2}^{*} g\right)\left(x_{1}, x_{2}\right) \mathcal{M} h\left(x_{1}, x_{2}\right),
\end{aligned}
$$


where $\int_{*}$ denotes $\iint_{\Omega_{2}}$. To estimate the remaining terms, we fix $r$ and $s$ such that $1 / q^{\prime}<1 / r<1 / q^{\prime}+\varepsilon$ and $1 / r^{\prime}=1 / s-\varepsilon$, where $1 / r+1 / r^{\prime}=1$. Then by the Hölder inequality and the Hardy-Littlewood-Sobolev theorem,

$$
\begin{aligned}
& \text { 12) } \quad\left|\iint_{\Omega_{2}} \eta_{9}\left(y_{1}, y_{2}\right) h\left(y_{1}, y_{2}\right) d y_{1} d y_{2}\right| \\
& \leq \frac{c}{t_{2}^{1+\varepsilon}} \int_{\left|y_{2}-x_{2}\right| \leq 4 t_{2}} M^{(1)} h\left(x_{1}, y_{2}\right) I_{\varepsilon}^{(2)}\left(M^{(1)}(g) \phi^{\prime}\left(\left|x_{2}-\cdot\right| / 16 t_{2}\right)\right)\left(x_{1}, y_{2}\right) d y_{2} \\
& \leq \frac{c}{t_{2}^{1+\varepsilon}}\left(\int_{\left|y_{2}-x_{2}\right| \leq 4 t_{2}}\left|M^{(1)} h\left(x_{1}, y_{2}\right)\right|^{r} d y_{2}\right)^{1 / r} \\
& \quad \times\left(\int_{\left|y_{2}-x_{2}\right| \leq 4 t_{2}}\left|I_{\varepsilon}^{(2)}\left(M^{(1)}(g) \phi^{\prime}\left(\left|x_{2}-\cdot\right| / 16 t_{2}\right)\right)\left(x_{1}, y_{2}\right)\right|^{r^{\prime}} d y_{2}\right)^{1 / r^{\prime}} \\
& \leq \frac{c}{t_{2}^{1+\varepsilon}}\left(\int_{\left|y_{2}-x_{2}\right| \leq 4 t_{2}}\left|M^{(1)} h\left(x_{1}, y_{2}\right)\right|^{r} d y_{2}\right)^{1 / r} \\
& \quad \times\left(\int\left|M^{(1)}(g)\left(x_{1}, y_{2}\right) \phi^{\prime}\left(\left|x_{2}-y_{2}\right| / 16 t_{2}\right)\right|^{s} d y_{2}\right)^{1 / s} \\
& \leq c M_{r}^{(2)}\left(M^{(1)} h\right)\left(x_{1}, x_{2}\right) M_{s}^{(2)}\left(M^{(1)} g\right)\left(x_{1}, x_{2}\right)
\end{aligned}
$$

and

$$
\begin{aligned}
& \left|\iint_{\Omega_{2}} \eta_{10}\left(y_{1}, y_{2}\right) h\left(y_{1}, y_{2}\right) d y_{1} d y_{2}\right| \\
\leq & \frac{c}{t_{2}^{1+\varepsilon}} \int_{\left|y_{2}-x_{2}\right| \leq 4 t_{2}} M^{(1)} h\left(x_{1}, y_{2}\right) I_{\varepsilon}^{(2)}\left(\widetilde{H}_{1}^{*}(g) \phi^{\prime}\left(\left|x_{2}-\cdot\right| / 16 t_{2}\right)\right)\left(x_{1}, y_{2}\right) d y_{2} \\
\leq & \frac{c}{t_{2}^{1+\varepsilon}}\left(\int_{\left|y_{2}-x_{2}\right| \leq 4 t_{2}}\left|M^{(1)} h\left(x_{1}, y_{2}\right)\right|^{r} d y_{2}\right)^{1 / r} \\
& \times\left(\int\left|\widetilde{H}_{1}^{*}(g)\left(x_{1}, y_{2}\right) \phi^{\prime}\left(\left|x_{2}-y_{2}\right| / 16 t_{2}\right)\right|^{s} d y_{2}\right)^{1 / s} \\
\leq & c M_{r}^{(2)}\left(M^{(1)} h\right)\left(x_{1}, x_{2}\right) M_{s}^{(2)}\left(\widetilde{H}_{1}^{*} g\right)\left(x_{1}, x_{2}\right) .
\end{aligned}
$$

Similarly, we obtain estimates for the last three terms:

$$
\begin{aligned}
& \left|\iint_{\Omega_{2}} \eta_{11}\left(y_{1}, y_{2}\right) h\left(y_{1}, y_{2}\right) d y_{1} d y_{2}\right| \\
& \leq c M_{r}^{(1)}\left(M^{(2)} h\right)\left(x_{1}, x_{2}\right) M_{s}^{(1)}\left(M^{(2)} g\right)\left(x_{1}, x_{2}\right) \\
& \left|\iint_{\Omega_{2}} \eta_{12}\left(y_{1}, y_{2}\right) h\left(y_{1}, y_{2}\right) d y_{1} d y_{2}\right| \\
& \leq c M_{r}^{(1)}\left(M^{(2)} h\right)\left(x_{1}, x_{2}\right) M_{s}^{(1)}\left(\widetilde{H}_{2}^{*} g\right)\left(x_{1}, x_{2}\right)
\end{aligned}
$$


and

(2.16)

$\left|\iint_{\Omega_{2}} \eta_{13}\left(y_{1}, y_{2}\right) h\left(y_{1}, y_{2}\right) d y_{1} d y_{2}\right|$

$\leq \frac{c}{t_{1}^{1+\varepsilon} t_{2}^{1+\varepsilon}} \int_{\left|y_{2}-x_{2}\right| \leq 4 t_{2}}\left(\int_{\left|y_{1}-x_{1}\right| \leq 4 t_{1}}\left|h\left(y_{1}, y_{2}\right)\right|^{r} d y_{1}\right)^{1 / r} \times$

$\left(\int_{\left|y_{1}-x_{1}\right| \leq 4 t_{1}}\left|I_{\varepsilon}^{(1)}\left(I_{\varepsilon}^{(2)}\left(|g| \phi^{\prime}\left(\left|x_{2}-\cdot\right| / 16 t_{2}\right)\right) \phi^{\prime}\left(\left|x_{1}-\cdot\right| / 16 t_{1}\right)\right)\left(y_{1}, y_{2}\right)\right|^{r^{\prime}} d y_{1}\right)^{1 / r^{\prime}} d y_{2}$ $\left|y_{1}-x_{1}\right| \leq 4 t_{1}$

$\leq \frac{c}{t_{1}^{1+\varepsilon} t_{2}^{1+\varepsilon}} \int_{\left|y_{2}-x_{2}\right| \leq 4 t_{2}}\left(\int_{\left|y_{1}-x_{1}\right| \leq 4 t_{1}}\left|h\left(y_{1}, y_{2}\right)\right|^{r} d y_{1}\right)^{1 / r}$

$\times\left(\int_{\left|y_{1}-x_{1}\right| \leq 16 t_{1}}\left|I_{\varepsilon}^{(2)}\left(|g| \phi^{\prime}\left(\left|x_{2}-\cdot\right| / 16 t_{2}\right)\right)\left(y_{1}, y_{2}\right)\right|^{s} d y_{1}\right)^{1 / s} d y_{2}$

$\leq \frac{c}{t_{1}^{1+\varepsilon} t_{2}^{1+\varepsilon}}\left(\iint_{\Omega_{2}}\left|h\left(y_{1}, y_{2}\right)\right|^{r} d y_{1}\right)^{1 / r}$

$\times\left(\int_{\left|y_{2}-x_{2}\right| \leq 4 t_{2}}\left(\int_{\left|y_{1}-x_{1}\right| \leq 16 t_{1}}\left|I_{\varepsilon}^{(2)}\left(|g| \phi^{\prime}\left(\left|x_{2}-\cdot\right| / 16 t_{2}\right)\right)\left(y_{1}, y_{2}\right)\right|^{s} d y_{1}\right)^{r^{\prime} / s} d y_{2}\right)^{1 / r^{\prime}}$

$\leq \frac{c}{t_{1}^{1+\varepsilon} t_{2}^{1+\varepsilon}}\left(\iint_{\Omega_{2}}\left|h\left(y_{1}, y_{2}\right)\right|^{r} d y_{1}\right)^{1 / r}$

$\times\left(\int_{\left|y_{1}-x_{1}\right| \leq 16 t_{1}}\left(\int_{\left|y_{2}-x_{2}\right| \leq 4 t_{2}}\left|I_{\varepsilon}^{(2)}\left(|g| \phi^{\prime}\left(\left|x_{2}-\cdot\right| / 16 t_{2}\right)\right)\left(y_{1}, y_{2}\right)\right|^{r^{\prime}} d y_{2}\right)^{s / r^{\prime}} d y_{1}\right)^{1 / s}$

$\leq \frac{c}{t_{1}^{1+\varepsilon} t_{2}^{1+\varepsilon}}\left(\iint_{\Omega_{2}}\left|h\left(y_{1}, y_{2}\right)\right|^{r} d y_{1}\right)^{1 / r}$

$\times\left(\int_{\left|y_{1}-x_{1}\right| \leq 16 t_{1}} \int_{\left|y_{2}-x_{2}\right| \leq 16 t_{2}}\left|g\left(y_{1}, y_{2}\right)\right|^{s} d y_{2} d y_{1}\right)^{1 / s}$

$\leq c M_{r}^{(1)}\left(M_{r}^{(2)} h\right)\left(x_{1}, x_{2}\right) M_{s}^{(1)}\left(M_{s}^{(2)} g\right)\left(x_{1}, x_{2}\right)$.

CASE III: $\left|x_{1}-y_{1}\right|>4 t_{1},\left|x_{2}-y_{2}\right| \leq 4 t_{2}$. Define

$$
\Omega_{3}=\left\{\left(y_{1}, y_{2}\right) \in \mathbb{R} \times \mathbb{R}:\left|x_{1}-y_{1}\right|>4 t_{1},\left|x_{2}-y_{2}\right| \leq 4 t_{2}\right\} .
$$

If $\left(y_{1}, y_{2}\right) \in \Omega_{3}$, then $\psi_{t_{1}}^{(1)}\left(x_{1}-y_{1}\right)=0$ and hence

$$
\begin{aligned}
& \iint \eta\left(y_{1}, y_{2}, z_{1}, z_{2}\right) d z_{1} d z_{2} \\
= & \iint \frac{g\left(z_{1}, z_{2}\right)}{\left(y_{1}-z_{1}\right)\left(y_{2}-z_{2}\right)} \psi_{t_{1}}^{(1)}\left(x_{1}-z_{1}\right)\left[\psi_{t_{2}}^{(2)}\left(x_{2}-z_{2}\right)-\psi_{t_{2}}^{(2)}\left(x_{2}-y_{2}\right)\right] d z_{1} d z_{2}
\end{aligned}
$$




$$
\begin{aligned}
& =\iint \frac{g\left(z_{1}, z_{2}\right)}{\left(y_{1}-z_{1}\right)\left(y_{2}-z_{2}\right)} \psi_{t_{1}}^{(1)}\left(x_{1}-z_{1}\right)\left[\psi_{t_{2}}^{(2)}\left(x_{2}-z_{2}\right)-\psi_{t_{2}}^{(2)}\left(x_{2}-y_{2}\right)\right] \\
& \times\left[\phi\left(\left|x_{2}-z_{2}\right| / 16 t_{2}\right)+\phi^{\prime}\left(\left|x_{2}-z_{2}\right| / 16 t_{2}\right)\right] d z_{1} d z_{2} \\
& =\left[-\psi_{t_{2}}^{(2)}\left(x_{2}-y_{2}\right)\right] \iint\left[\frac{1}{y_{1}-z_{1}}-\frac{1}{y_{1}-x_{1}}\right]\left[\frac{1}{y_{2}-z_{2}}-\frac{1}{x_{2}-z_{2}}\right] \\
& \times \psi_{t_{1}}^{(1)}\left(x_{1}-z_{1}\right) \phi\left(\left|x_{2}-z_{2}\right| / 16 t_{2}\right) g\left(z_{1}, z_{2}\right) d z_{1} d z_{2} \\
& +\left[-\psi_{t_{2}}^{(2)}\left(x_{2}-y_{2}\right)\right] \iint \frac{1}{y_{1}-x_{1}}\left[\frac{1}{y_{2}-z_{2}}-\frac{1}{x_{2}-z_{2}}\right] \\
& \times \psi_{t_{1}}^{(1)}\left(x_{1}-z_{1}\right) \phi\left(\left|x_{2}-z_{2}\right| / 16 t_{2}\right) g\left(z_{1}, z_{2}\right) d z_{1} d z_{2} \\
& +\left[-\psi_{t_{2}}^{(2)}\left(x_{2}-y_{2}\right)\right] \iint\left[\frac{1}{y_{1}-z_{1}}-\frac{1}{y_{1}-x_{1}}\right] \frac{1}{x_{2}-z_{2}} \\
& \times \psi_{t_{1}}^{(1)}\left(x_{1}-z_{1}\right) \phi\left(\left|x_{2}-z_{2}\right| / 16 t_{2}\right) g\left(z_{1}, z_{2}\right) d z_{1} d z_{2} \\
& +\left[-\psi_{t_{2}}^{(2)}\left(x_{2}-y_{2}\right)\right] \iint \frac{g\left(z_{1}, z_{2}\right)}{\left(y_{1}-x_{1}\right)\left(x_{2}-z_{2}\right)} \psi_{t_{1}}^{(1)}\left(x_{1}-z_{1}\right) \phi\left(\left|x_{2}-z_{2}\right| / 16 t_{2}\right) d z_{1} d z_{2} \\
& +\iint\left[\frac{1}{y_{1}-z_{1}}-\frac{1}{y_{1}-x_{1}}\right] \frac{1}{y_{2}-z_{2}} \psi_{t_{1}}^{(1)}\left(x_{1}-z_{1}\right)\left[\psi_{t_{2}}^{(2)}\left(x_{2}-z_{2}\right)-\psi_{t_{2}}^{(2)}\left(x_{2}-y_{2}\right)\right] \\
& \times \phi^{\prime}\left(\left|x_{2}-z_{2}\right| / 16 t_{2}\right) g\left(z_{1}, z_{2}\right) d z_{1} d z_{2} \\
& +\iint \frac{1}{\left(y_{1}-x_{1}\right)\left(y_{2}-z_{2}\right)} \psi_{t_{1}}^{(1)}\left(x_{1}-z_{1}\right)\left[\psi_{t_{2}}^{(2)}\left(x_{2}-z_{2}\right)-\psi_{t_{2}}^{(2)}\left(x_{2}-y_{2}\right)\right] \\
& \times \phi^{\prime}\left(\left|x_{2}-z_{2}\right| / 16 t_{2}\right) g\left(z_{1}, z_{2}\right) d z_{1} d z_{2} \\
& =\sum_{j=14}^{19} \eta_{j}\left(y_{1}, y_{2}\right)
\end{aligned}
$$

If $\psi_{t_{1}}^{(1)}\left(x_{1}-z_{1}\right) \neq 0$ then $\left|x_{1}-z_{1}\right| \leq t_{1}$ and $\left|x_{1}-y_{1}\right|>4 t_{1}$, hence $\left|x_{1}-z_{1}\right|<$ $\left|x_{1}-y_{1}\right| / 2$. Similarly, if $\phi\left(\left|x_{2}-z_{2}\right| / 16 t_{2}\right) \neq 0$, then $\left|x_{2}-y_{2}\right| \leq\left|x_{2}-z_{2}\right| / 2$. Thus

$$
\begin{aligned}
\left|\eta_{14}\left(y_{1}, y_{2}\right)\right| \leq & c\left|\psi_{t_{2}}^{(2)}\left(x_{2}-y_{2}\right)\right| \frac{t_{1}}{\left(t_{1}+\left|y_{1}-x_{1}\right|\right)^{2}} \\
& \quad \times \iint\left|\psi_{t_{1}}^{(1)}\left(x_{1}-z_{1}\right)\right| \frac{t_{2}}{\left(t_{2}+\left|x_{2}-z_{2}\right|\right)^{2}}\left|g\left(z_{1}, z_{2}\right)\right| d z_{1} d z_{2} \\
\leq & c\left|\psi_{t_{2}}^{(2)}\left(x_{2}-y_{2}\right)\right| \frac{t_{1}}{\left(t_{1}+\left|y_{1}-x_{1}\right|\right)^{2}} \mathcal{M g}\left(x_{1}, x_{2}\right) .
\end{aligned}
$$

Integrating $\eta_{14}\left(y_{1}, y_{2}\right)$ against $h\left(y_{1}, y_{2}\right)$ over $\Omega_{3}$ with respect to $y_{1}$ and $y_{2}$, we easily get 


$$
\left|\iint_{\Omega_{3}} \eta_{14}\left(y_{1}, y_{2}\right) h\left(y_{1}, y_{2}\right) d y_{1} d y_{2}\right| \leq c \mathcal{M} g\left(x_{1}, x_{2}\right) \mathcal{M} h\left(x_{1}, x_{2}\right) .
$$

To estimate $\eta_{15}\left(y_{1}, y_{2}\right)$, we integrate it against $h\left(y_{1}, y_{2}\right)$ over $\Omega_{3}$ directly:

$$
\begin{aligned}
& \left|\iint_{\Omega_{3}} \eta_{15}\left(y_{1}, y_{2}\right) h\left(y_{1}, y_{2}\right) d y_{1} d y_{2}\right| \\
& \leq c \mathcal{M g}\left(x_{1}, x_{2}\right) \int_{\left|y_{2}-x_{2}\right| \leq 4 t_{2}} H_{1}^{*} h\left(x_{1}, y_{2}\right)\left|\psi_{t_{2}}^{(2)}\left(x_{2}-y_{2}\right)\right| d y_{2} \\
& \leq c \mathcal{M g}\left(x_{1}, x_{2}\right) M^{(2)}\left(H_{1}^{*} h\right)\left(x_{1}, x_{2}\right) .
\end{aligned}
$$

Similarly, we obtain an estimate for $\eta_{16}\left(y_{1}, y_{2}\right)$ :

$$
\left|\eta_{16}\left(y_{1}, y_{2}\right)\right| \leq c\left|\psi_{t_{2}}^{(2)}\left(x_{2}-y_{2}\right)\right| \frac{t_{1}}{\left(t_{1}+\left|y_{1}-x_{1}\right|\right)^{2}} \widetilde{H}_{2}^{*}\left(M^{(1)} g\right)\left(x_{1}, x_{2}\right),
$$

which yields

$$
\left|\iint_{\Omega_{3}} \eta_{16}\left(y_{1}, y_{2}\right) h\left(y_{1}, y_{2}\right) d y_{1} d y_{2}\right| \leq c \widetilde{H}_{2}^{*}\left(M^{(1)} g\right)\left(x_{1}, x_{2}\right) \mathcal{M} h\left(x_{1}, x_{2}\right) .
$$

To estimate $\eta_{17}\left(y_{1}, y_{2}\right)$, we integrate it against $h\left(y_{1}, y_{2}\right)$ over $\Omega_{3}$ directly:

$$
\begin{aligned}
& \left|\iint_{\Omega_{3}} \eta_{17}\left(y_{1}, y_{2}\right) h\left(y_{1}, y_{2}\right) d y_{1} d y_{2}\right| \\
& \quad \leq c \widetilde{H}_{2}^{*}\left(M^{(1)} g\right)\left(x_{1}, x_{2}\right) \int_{\left|y_{2}-x_{2}\right| \leq 4 t_{2}} H_{1}^{*} h\left(x_{1}, y_{2}\right)\left|\psi_{t_{2}}^{(2)}\left(x_{2}-y_{2}\right)\right| d y_{2} \\
& \quad \leq c \widetilde{H}_{2}^{*}\left(M^{(1)} g\right)\left(x_{1}, x_{2}\right) M^{(2)}\left(H_{1}^{*} h\right)\left(x_{1}, x_{2}\right) .
\end{aligned}
$$

To estimate $\eta_{18}\left(y_{1}, y_{2}\right)$ and $\eta_{19}\left(y_{1}, y_{2}\right)$, we use the Lipschitz continuity of $\psi_{t_{1}}^{(1)}$ and $\psi_{t_{2}}^{(2)}$ :

$\left|\eta_{18}\left(y_{1}, y_{2}\right)\right|$

$$
\begin{aligned}
& \leq c \frac{t_{1}}{\left(t_{1}+\left|y_{1}-x_{1}\right|\right)^{2}} \iint \frac{\left|\psi_{t_{1}}^{(1)}\left(x_{1}-z_{1}\right)\right|}{t_{2}^{1+\varepsilon}} \frac{\phi^{\prime}\left(\left|x_{2}-z_{2}\right| / 16 t_{2}\right)}{\left|y_{2}-z_{2}\right|^{1-\varepsilon}}\left|g\left(z_{1}, z_{2}\right)\right| d z_{1} d z_{2} \\
& \leq c \frac{t_{1}}{\left(t_{1}+\left|y_{1}-x_{1}\right|\right)^{2}} \frac{1}{t_{2}^{1+\varepsilon}} I_{\varepsilon}^{(2)}\left(M^{(1)}(g) \phi^{\prime}\left(\left|x_{2}-\cdot\right| / 16 t_{2}\right)\right)\left(x_{1}, y_{2}\right) .
\end{aligned}
$$

By the Hölder inequality and the Hardy-Littlewood-Sobolev theorem,

$$
\begin{aligned}
& \quad\left|\iint_{\Omega_{3}} \eta_{18}\left(y_{1}, y_{2}\right) h\left(y_{1}, y_{2}\right) d y_{1} d y_{2}\right| \\
& \leq \frac{c}{t_{2}^{1+\varepsilon}} \int_{\left|y_{2}-x_{2}\right| \leq 4 t_{2}} M^{(1)} h\left(x_{1}, y_{2}\right) I_{\varepsilon}^{(2)}\left(M^{(1)}(g) \phi^{\prime}\left(\left|x_{2}-\cdot\right| / 16 t_{2}\right)\right)\left(x_{1}, y_{2}\right) d y_{2}
\end{aligned}
$$




$$
\begin{aligned}
\leq & \frac{c}{t_{2}^{1+\varepsilon}}\left(\int_{\left|y_{2}-x_{2}\right| \leq 4 t_{2}}\left|M^{(1)} h\left(x_{1}, y_{2}\right)\right|^{r} d y_{2}\right)^{1 / r} \\
& \times\left(\int_{\left|y_{2}-x_{2}\right| \leq 4 t_{2}}\left|I_{\varepsilon}^{(2)}\left(M^{(1)}(g) \phi^{\prime}\left(\left|x_{2}-\cdot\right| / 16 t_{2}\right)\right)\left(x_{1}, y_{2}\right)\right|^{r^{\prime}} d y_{2}\right)^{1 / r^{\prime}} \\
\leq & \frac{c}{t_{2}^{1+\varepsilon}}\left(\int_{\left|y_{2}-x_{2}\right| \leq 4 t_{2}}\left|M^{(1)} h\left(x_{1}, y_{2}\right)\right|^{r} d y_{2}\right)^{1 / r} \\
& \times\left(\int\left|M^{(1)}(g)\left(x_{1}, y_{2}\right) \phi^{\prime}\left(\left|x_{2}-y_{2}\right| / 16 t_{2}\right)\right|^{s} d y_{2}\right)^{1 / s} \\
\leq & c M_{r}^{(2)}\left(M^{(1)} h\right)\left(x_{1}, x_{2}\right) M_{s}^{(2)}\left(M^{(1)} g\right)\left(x_{1}, x_{2}\right) .
\end{aligned}
$$

To estimate $\eta_{19}\left(y_{1}, y_{2}\right)$, using a method similar to that for $\eta_{18}\left(y_{1}, y_{2}\right)$, we integrate it against $h\left(y_{1}, y_{2}\right)$ over $\Omega_{3}$ directly to obtain

$$
\left|\iint_{\Omega_{3}} \eta_{19}\left(y_{1}, y_{2}\right) h\left(y_{1}, y_{2}\right) d y_{1} d y_{2}\right|
$$

$$
\begin{aligned}
& \leq \frac{c}{t_{2}^{1+\varepsilon}} \int_{\left|y_{2}-x_{2}\right| \leq 4 t_{2}} H_{1}^{*} h\left(x_{1}, y_{2}\right)\left(\int \frac{\phi^{\prime}\left(\left|x_{2}-z_{2}\right| / 16 t_{2}\right)}{\left|y_{2}-z_{2}\right|^{1-\varepsilon}} M^{(1)} g\left(x_{1}, z_{2}\right) d z_{2}\right) d y_{2} \\
& \leq \frac{c}{t_{2}^{1+\varepsilon}} \int_{\left|y_{2}-x_{2}\right| \leq 4 t_{2}} H_{1}^{*} h\left(x_{1}, y_{2}\right) I_{\varepsilon}^{(2)}\left(M^{(1)}(g) \phi^{\prime}\left(\left|x_{2}-\cdot\right| / 16 t_{2}\right)\right)\left(x_{1}, y_{2}\right) d y_{2} \\
& \leq c M_{r}^{(2)}\left(H_{1}^{*} h\right)\left(x_{1}, x_{2}\right) M_{s}^{(2)}\left(M^{(1)} g\right)\left(x_{1}, x_{2}\right) .
\end{aligned}
$$

CASE IV: $\left|x_{1}-y_{1}\right| \leq 4 t_{1},\left|x_{2}-y_{2}\right|>4 t_{2}$. Define

$$
\Omega_{4}=\left\{\left(y_{1}, y_{2}\right) \in \mathbb{R} \times \mathbb{R}:\left|x_{1}-y_{1}\right| \leq 4 t_{1},\left|x_{2}-y_{2}\right|>4 t_{2}\right\} .
$$

The estimates in this case are similar to those of Case III. We omit the details.

By (2.1), we have

$$
\begin{aligned}
\iint D(g, h)\left(y_{1}, y_{2}\right) \psi_{t_{1}}^{(1)} & \left(x_{1}-y_{1}\right) \psi_{t_{2}}^{(2)}\left(x_{2}-y_{2}\right) d y_{1} d y_{2} \\
= & \iint \eta\left(y_{1}, y_{2}, z_{1}, z_{2}\right) d z_{1} d z_{2} h\left(y_{1}, y_{2}\right) d y_{1} d y_{2} \\
= & \iint_{\Omega_{1}} \eta\left(y_{1}, y_{2}, z_{1}, z_{2}\right) d z_{1} d z_{2} h\left(y_{1}, y_{2}\right) d y_{1} d y_{2} \\
& +\iint_{\Omega_{2}} \eta\left(y_{1}, y_{2}, z_{1}, z_{2}\right) d z_{1} d z_{2} h\left(y_{1}, y_{2}\right) d y_{1} d y_{2} \\
& +\iint_{\Omega_{3}} \eta\left(y_{1}, y_{2}, z_{1}, z_{2}\right) d z_{1} d z_{2} h\left(y_{1}, y_{2}\right) d y_{1} d y_{2}
\end{aligned}
$$




$$
\begin{aligned}
& +\iint_{\Omega_{4}} \eta\left(y_{1}, y_{2}, z_{1}, z_{2}\right) d z_{1} d z_{2} h\left(y_{1}, y_{2}\right) d y_{1} d y_{2} \\
= & I\left(x_{1}, x_{2}\right)+I I\left(x_{1}, x_{2}\right)+I I I\left(x_{1}, x_{2}\right)+I V\left(x_{1}, x_{2}\right) .
\end{aligned}
$$

By $(2.2)-(2.6)$, we get

$$
\begin{aligned}
\left|I\left(x_{1}, x_{2}\right)\right| \leq & c \mathcal{M g}\left(x_{1}, x_{2}\right)\left\{\mathcal{M} h\left(x_{1}, x_{2}\right)+M^{(2)}\left(H_{1}^{*} h\right)\left(x_{1}, x_{2}\right)\right. \\
& \left.+M^{(1)}\left(H_{2}^{*} h\right)\left(x_{1}, x_{2}\right)+H_{1}^{*}\left(H_{2}^{*} h\right)\left(x_{1}, x_{2}\right)\right\}
\end{aligned}
$$

Corresponding to $(2.7), I I\left(x_{1}, x_{2}\right)$ consists of nine terms. By the estimates (2.8)-(2.16), we obtain an estimate for $I I\left(x_{1}, x_{2}\right)$ :

$$
\begin{aligned}
& \left|I I\left(x_{1}, x_{2}\right)\right| \leq c \mathcal{M h}\left(x_{1}, x_{2}\right)\left\{\mathcal{M} g\left(x_{1}, x_{2}\right)+M^{(2)}\left(\widetilde{H}_{1}^{*} g\right)\left(x_{1}, x_{2}\right)\right. \\
+ & \left.M^{(1)}\left(\widetilde{H}_{2}^{*} g\right)\left(x_{1}, x_{2}\right)+\widetilde{H}_{1}^{*}\left(H_{2}^{*} g\right)\left(x_{1}, x_{2}\right)\right\} \\
+ & c M_{r}^{(2)}\left(M^{(1)} h\right)\left(x_{1}, x_{2}\right)\left\{M_{s}^{(2)}\left(M^{(1)} g\right)\left(x_{1}, x_{2}\right)+M_{s}^{(2)}\left(\widetilde{H}_{1}^{*} g\right)\left(x_{1}, x_{2}\right)\right\} \\
+ & c M_{r}^{(1)}\left(M^{(2)} h\right)\left(x_{1}, x_{2}\right)\left\{M_{s}^{(1)}\left(M^{(2)} g\right)\left(x_{1}, x_{2}\right)+M_{s}^{(1)}\left(\widetilde{H}_{2}^{*} g\right)\left(x_{1}, x_{2}\right)\right\} \\
+ & c M_{r}^{(1)}\left(M_{r}^{(2)} h\right)\left(x_{1}, x_{2}\right) M_{s}^{(1)}\left(M_{s}^{(2)} g\right)\left(x_{1}, x_{2}\right) .
\end{aligned}
$$

Corresponding to $(2.17), I I I\left(x_{1}, x_{2}\right)$ has six terms. Collecting the estimates (2.18)-(2.23) for $I I I\left(x_{1}, x_{2}\right)$, we have

$$
\begin{aligned}
& \left|I I I\left(x_{1}, x_{2}\right)\right| \leq c \mathcal{M} g\left(x_{1}, x_{2}\right)\left\{\mathcal{M} h\left(x_{1}, x_{2}\right)+M^{(2)}\left(H_{1}^{*} h\right)\left(x_{1}, x_{2}\right)\right\} \\
+ & c \widetilde{H}_{2}^{*}\left(M^{(1)} g\right)\left(x_{1}, x_{2}\right)\left\{\mathcal{M} h\left(x_{1}, x_{2}\right)+M^{(2)}\left(H_{1}^{*} h\right)\left(x_{1}, x_{2}\right)\right\} \\
+ & c M_{s}^{(2)}\left(M^{(1)} g\right)\left(x_{1}, x_{2}\right)\left\{M_{r}^{(2)}\left(M^{(1)} h\right)\left(x_{1}, x_{2}\right)+M_{r}^{(2)}\left(H_{1}^{*} h\right)\left(x_{1}, x_{2}\right)\right\} .
\end{aligned}
$$

The same argument gives estimates for $I V\left(x_{1}, x_{2}\right)$. Taking the supremum in $(2.24)$ for $t_{1}, t_{2}>0$ together with $(2.25)-(2.27)$, we obtain a maximal function estimate for the bilinear form:

$$
\begin{aligned}
& {[D(g, h)]^{*}\left(x_{1}, x_{2}\right) \leq c \mathcal{M g}\left(x_{1}, x_{2}\right)\left\{\mathcal{M} h\left(x_{1}, x_{2}\right)+M^{(2)}\left(H_{1}^{*} h\right)\left(x_{1}, x_{2}\right)\right.} \\
& \left.+M^{(1)}\left(H_{2}^{*} h\right)\left(x_{1}, x_{2}\right)+H_{1}^{*}\left(H_{2}^{*} h\right)\left(x_{1}, x_{2}\right)\right\} \\
& +c \mathcal{M h}\left(x_{1}, x_{2}\right)\left\{\mathcal{M} g\left(x_{1}, x_{2}\right)+M^{(2)}\left(\widetilde{H}_{1}^{*} g\right)\left(x_{1}, x_{2}\right)\right. \\
& \left.+M^{(1)}\left(\widetilde{H}_{2}^{*} g\right)\left(x_{1}, x_{2}\right)+\widetilde{H}_{1}^{*}\left(H_{2}^{*} g\right)\left(x_{1}, x_{2}\right)\right\} \\
& +c M_{r}^{(2)}\left(M^{(1)} h\right)\left(x_{1}, x_{2}\right)\left\{M_{s}^{(2)}\left(M^{(1)} g\right)\left(x_{1}, x_{2}\right)+M_{s}^{(2)}\left(\widetilde{H}_{1}^{*} g\right)\left(x_{1}, x_{2}\right)\right\} \\
& +c M_{r}^{(1)}\left(M^{(2)} h\right)\left(x_{1}, x_{2}\right)\left\{M_{s}^{(1)}\left(M^{(2)} g\right)\left(x_{1}, x_{2}\right)+M_{s}^{(1)}\left(\widetilde{H}_{2}^{*} g\right)\left(x_{1}, x_{2}\right)\right\} \\
& +c M_{r}^{(1)}\left(M_{r}^{(2)} h\right)\left(x_{1}, x_{2}\right) M_{s}^{(1)}\left(M_{s}^{(2)} g\right)\left(x_{1}, x_{2}\right) \\
& +c \widetilde{H}_{2}^{*}\left(M^{(1)} g\right)\left(x_{1}, x_{2}\right)\left\{\mathcal{M} h\left(x_{1}, x_{2}\right)+M^{(2)}\left(H_{1}^{*} h\right)\left(x_{1}, x_{2}\right)\right\} \\
& +c M_{s}^{(2)}\left(M^{(1)} g\right)\left(x_{1}, x_{2}\right)\left\{M_{r}^{(2)}\left(M^{(1)} h\right)\left(x_{1}, x_{2}\right)+M_{r}^{(2)}\left(H_{1}^{*} h\right)\left(x_{1}, x_{2}\right)\right\} \\
& \text { + other terms corresponding to Case IV. }
\end{aligned}
$$


We now show that all terms on the right hand side belong to $L^{1}(\mathbb{R} \times \mathbb{R})$. By the boundedness of the maximal functions and the maximal operators of the Hilbert transforms $H_{1}$ and $H_{2}$, we can easily see that the $L^{1}(\mathbb{R} \times \mathbb{R})$ norm of the first two terms is controlled by $c\|g\|_{L^{q}(\mathbb{R} \times \mathbb{R})}\|h\|_{L^{q^{\prime}}(\mathbb{R} \times \mathbb{R})}$.

For the fifth term on the right hand side, we have $s<q$ and $r<q^{\prime}$ by the choice of $r$ and $s$. So by the Hölder inequality,

$$
\begin{aligned}
& \iint M_{r}^{(1)}\left(M_{r}^{(2)} h\right)\left(x_{1}, x_{2}\right) M_{s}^{(1)}\left(M_{s}^{(2)} g\right)\left(x_{1}, x_{2}\right) d x_{1} d x_{2} \\
& \leq \int_{\mathbb{R}}\left(\int_{\mathbb{R}}\left|M_{r}^{(1)}\left(M_{r}^{(2)} h\right)\left(x_{1}, x_{2}\right)\right|^{q^{\prime}} d x_{1}\right)^{1 / q^{\prime}}\left(\int_{\mathbb{R}}\left|M_{s}^{(1)}\left(M_{s}^{(2)} g\right)\left(x_{1}, x_{2}\right)\right|^{q} d x_{1}\right)^{1 / q} d x_{2} \\
& \left.\leq\left. c \int_{\mathbb{R}}\left(\int_{\mathbb{R}}\left|M_{r}^{(2)} h\left(x_{1}, x_{2}\right)\right|^{q^{\prime}} d x_{1}\right)^{1 / q^{\prime}}\left(\int_{\mathbb{R}} \mid M_{s}^{(2)} g\right)\left(x_{1}, x_{2}\right)\right|^{q} d x_{1}\right)^{1 / q} d x_{2} \\
& \leq c\left(\iint\left|M_{r}^{(2)} h\left(x_{1}, x_{2}\right)\right|^{q^{\prime}} d x_{1} d x_{2}\right)^{1 / q^{\prime}}\left(\iint\left|M_{s}^{(2)} g\left(x_{1}, x_{2}\right)\right|^{q} d x_{1} d x_{2}\right)^{1 / q} \\
& \leq c\left(\iint\left|h\left(x_{1}, x_{2}\right)\right|^{q^{\prime}} d x_{1} d x_{2}\right)^{1 / q^{\prime}}\left(\iint\left|g\left(x_{1}, x_{2}\right)\right|^{q} d x_{1} d x_{2}\right)^{1 / q} \\
& \leq c\|g\|_{L^{q}(\mathbb{R} \times \mathbb{R})}\|h\|_{L^{q^{\prime}}(\mathbb{R} \times \mathbb{R})} .
\end{aligned}
$$

The other terms can be dealt with similarly. Hence we finally obtain

$$
\iint[D(g, h)]^{*}\left(x_{1}, x_{2}\right) d x_{1} d x_{2} \leq c\|g\|_{L^{q}(\mathbb{R} \times \mathbb{R})}\|h\|_{L^{q^{\prime}(\mathbb{R} \times \mathbb{R})}} .
$$

This completes the proof of Theorem 1.

From Theorem 1, we can easily deduce Theorem 2 as follows.

Proof of Theorem 2. To obtain the factorization theorem, consider the Banach space

$$
B=\left\{f \in L^{1}(\mathbb{R} \times \mathbb{R}): f=\sum_{i=1}^{\infty} D\left(g_{i}, h_{i}\right), g_{i}, h_{i} \in L^{2}(\mathbb{R} \times \mathbb{R}), i=1,2, \ldots\right\}
$$

and define

$$
\|f\|_{B}=\inf \sum_{i=1}^{\infty}\left\|g_{i}\right\|_{2}\left\|h_{i}\right\|_{2}
$$

where the infimum is taken over all decompositions of $f$.

By Theorem 1,

$$
\|f\|_{H^{1}} \leq \sum_{i=1}^{\infty}\left\|D\left(g_{i}, h_{i}\right)\right\|_{H^{1}} \leq c \sum_{i=1}^{\infty}\left\|g_{i}\right\|_{2}\left\|h_{i}\right\|_{2}
$$

So, $B$ is a subspace of $H^{1}(\mathbb{R} \times \mathbb{R})$. On the other hand, if $b$ is in BMO, then formally

$$
\langle b, D(g, h)\rangle=\left\langle\left[\left[b, H_{1}\right], H_{2}\right](g), h\right\rangle .
$$


By Theorem 1.2 in [5],

$$
\|b\|_{\mathrm{BMO}} \cong\left\|\left[\left[b, H_{1}\right], H_{2}\right]\right\|_{L^{2} \rightarrow L^{2}} \cong \sup \left\{\left|\iint f b\right|:\|f\|_{B}=1\right\} .
$$

This gives the equivalence of the $H^{1}$ norm and $\|\cdot\|_{B}$, showing that the two spaces are the same. The proof is complete.

\section{References}

[1] S. A. Chang and R. Fefferman, A continuous version of the duality of $H^{1}$ and BMO on the bi-disc, Ann. of Math. 112 (1980), 179-201.

[2] - - - Some recent developments in Fourier analysis and $H^{p}$ theory on product domains, Bull. Amer. Math. Soc. 12 (1985), 1-43.

[3] R. R. Coifman, R. Rochberg and G. Weiss, Factorization theorems for Hardy spaces in several variables, Ann. of Math. 103 (1976), 611-635.

[4] R. Fefferman, Calderón-Zygmund theory for product domains: $H^{p}$ spaces, Proc. Natl. Acad. Sci. USA 83 (1986), 840-843.

[5] S. H. Ferguson and M. T. Lacey, A characterization of product BMO by commutators, Acta Math. 189 (2002), 143-160.

[6] S. H. Ferguson and C. Sadosky, Characterizations of bounded mean oscillation on the polydisk in terms of Hankel operators and Carleson measures, J. Anal. Math. 81 (2000), 239-267.

[7] A. Uchiyama, The factorization of $H^{p}$ on the space of homogeneous type, Pacific J. Math. 92 (1981), 453-468.

Institute of Applied Physics and Computational Mathematics

P.O. Box 8009, Beijing, 100088

People's Republic of China

E-mail: chenwg@iapcm.ac.cn miao_changxing@iapcm.ac.cn
Department of Mathematics Auburn University Auburn, AL 36849-5310, U.S.A. E-mail: hanyong@mail.auburn.edu

Received July 29, 2005

Revised version October 8, 2006 